\title{
Glucosylceramide synthase regulates the proliferation and apoptosis of liver cells in vitro by Bcl-2/Bax pathway
}

\author{
JUN-FENG LI ${ }^{1,2^{*}}$, SU-JUN ZHENG ${ }^{3 *}$, LI-LI WANG ${ }^{1,4 *}$, SHUANG LIU $^{3}$, FENG REN $^{5}$, \\ $\mathrm{YU} \mathrm{CHEN}^{3}$, LI BAI ${ }^{3}$, MEI LIU ${ }^{3}$ and ZHONG-PING DUAN ${ }^{3}$
}

\begin{abstract}
${ }^{1}$ The First Clinical Medical School of Lanzhou University; ${ }^{2}$ Institute of Infectious Diseases, Department of Infectious Diseases, The First Hospital of Lanzhou University, Lanzhou, Gansu $730000 ;{ }^{3}$ Artificial Liver Center, Beijing YouAn Hospital, Capital Medical University, Beijing 100069; ${ }^{4}$ Department of Radiology, The First Hospital of Lanzhou University, Lanzhou, Gansu 730000; ${ }^{5}$ Institute of Liver Diseases, Beijing YouAn Hospital, Capital Medical University, Beijing 100069, P.R. China
\end{abstract}

Received April 27, 2017; Accepted September 7, 2017

DOI: $10.3892 / \mathrm{mmr} .2017 .7580$

\begin{abstract}
Our previous study found that glucosylceramide, a type of sphingolipids, was associated with liver inflammation and fibrosis. Glucosylceramide is generated by glucosylceramide synthase (GCS), which is encoded by the UDP-glucose ceramide glucosyltransferase (UGCG) gene. GCS is a key enzyme to regulate the physiological activity of cells. However, the role of GCS in hepatic cells remains unclear. The aim of the present study was to explore the mechanism of GCS in the proliferation and apoptosis of liver cells. Following the interference of expression of GCS in vitro by UGCG small interfering (si)RNA, the MTT method was performed to detect the proliferation of HL-7702 hepatocytes, and ELISA was used to determine the concentration of tumor necrosis factor (TNF) $\alpha$ and cytochrome $c$ in the supernatant of culture system. Fluorescence microscopy was used to observe the apoptosis of liver cells stained by Annexin V-fluorescein isothiocyanate/propidium iodide. Reverse transcription-quantitative polymerase chain reaction was used to detect the gene expression apoptosis regulator $\mathrm{Bcl}-2$ (Bcl-2), apoptosis regulator Bax (Bax) and caspase-3. Western blot analysis was used to detect the expression of caspase-3 protein in the liver cells. Following treatment with UGCG siRNA for $24 \mathrm{~h}$, the proliferation of HL-7702 hepatocytes was significantly inhibited when compared with the transfection reagent group. Furthermore, the early and advanced apoptosis of liver cells showed an increasing trend. Additionally, concentrations of
\end{abstract}

Correspondence to: Dr Zhong-Ping Duan, Artificial Liver Center, Beijing YouAn Hospital, Capital Medical University, 8 Xitoutiao, Youwai Street, Beijing 100069, P.R. China

E-mail: duan2517@163.com

*Contributed equally

Key words: sphingolipids, glucosylceramide synthase, hepatocytes, proliferation, apoptosis
TNF $\alpha$ and cytochrome $c$ showed no significant difference between the UGCG siRNA and transfection reagent groups. Compared with the transfection reagent group, Bcl-2 mRNA expression decreased, and Bax and caspase-3 mRNA expression increased in the UGCG siRNA transfection group. The protein expression level of caspase-3 showed increased in hepatocytes following the treatment with UGCG siRNA. In conclusion, the metabolic changes of sphingolipids caused by the lack of GCS may be involved in the proliferation and apoptosis of liver cells through the Bcl-2/Bax signaling pathway.

\section{Introduction}

Chronic liver injury, playing a key role in the pathogenesis of cirrhosis, liver failure or hepatocellular carcinoma can be caused by the infection, metabolism, alcohol and drugs $(1,2)$. It has been confirmed that the apoptosis of hepatocyte induced by immune response in chronic liver injury is one of the main reasons for the onset or development of liver diseases such as viral hepatitis, autoimmune liver disease, post-orthotopic liver transplantation, alcoholic liver disease, drug-induced liver injury and non-alcoholic fatty liver (3). The inhibition of hepatocyte apoptosis in the process of liver injury becomes an important strategy for the treatment of liver diseases clinically.

Sphingolipids are enriched in lipid rafts of cellular membranes and contribute to important physiological cellular processes including cell proliferation, differentiation and apoptosis (4). Recently, it has been confirmed that sphingolipids play roles in regulating liver injury and regeneration, which may have a great impact on the development of novel therapeutic modalities for a variety of liver diseases (5). Our previously series of studies showed that sphingolipids and their metabolism played important roles in the development of liver diseases (6-10). Based on our results, we have found that the increase of plasma hexosylceramide in chronic hepatitis $\mathrm{C}$ patients significantly correlated with hepatic necroinflammation, and it showed a close relationship with liver inflammation or fibrosis. Glycosphingolipids include a family of heterogeneous lipids which regulate cell death pathways through mitochondria, or endoplasmic reticulum to mediate apoptosis, 
endoplasmic reticulum stress, autophagy, and necroptosis (11). Hexosylceramide is formed by glycosylation of ceramide caused by glucosylceramide synthase (GCS) which linked glucose to 1-hydroxy group of ceramide. In the metabolism process of glycosphingolipids, GCS which is encoded by the UDP-glucose ceramide glucosyltransferase (UGCG) gene, is a key enzyme catalyzing glycosylation of ceramide to regulate the physiological activity of cells by influencing the metabolic balance of ceramide and glycosphingolipids (12). However the detailed mechanisms of the glycosphingolipids' metabolism in the proliferation and apoptosis of liver cells remained poorly understood.

Therefore, on the basis of our previous studies, the present study speculated that there might be a close relationship between GCS and the proliferation or apoptosis of liver cells. This study was aimed to evaluate the role of GCS in the proliferation and apoptosis of liver cells and explore the underlying mechanisms.

\section{Materials and methods}

Cell culture. Human liver cells line HL-7702 was a persevered cell line in Artificial Liver Center at Beijing You'an Hospital, Capital Medical University (Beijing, China). The cells were cultured in RPMI-1640 medium containing 10\% fetal bovine serum (both from Hyclone, Logan, UT, USA) in $37^{\circ} \mathrm{C}$ and $5 \% \mathrm{CO}_{2}$ cell culture incubator (Thermo Fisher Scientific, Inc., Waltham, MA, USA). The medium was changed every other day after plating and the cells were digested with $0.05 \%$ trypsin when they were $80-90 \%$ confluent.

siRNA transfection. The HL-7702 cells were transfected with siRNA when the cells were $80 \%$ confluent. The siRNA and Lipofectamine 2000 (Invitrogen; Thermo Fisher Scientific, Inc.) were diluted separately and mixed according to the product instructions. The serum-free medium containing siRNA-Lipofectamin 2000 (the concentration of siRNA: $0.12 \mu \mathrm{mol} / \mathrm{l}$ ) was left to incubate for $25 \mathrm{~min}$ at room temperature, and then the siRNA-Lipofectamin 2000 mix was put into the culture medium of the adherent cells.

Proliferation assay. The cells were added into 96-well plate (Corning Incorporated, Corning, NY, USA) as 8,000 cells/well with RPMI-1640 medium containing 10\% fetal bovine serum (both from Hyclone). After that the cells were $80 \%$ confluent, the medium was without fetal bovine serum in the blank control group. The cells with Lipofectamin 2000 (Invitrogen; Thermo Fisher Scientific, Inc.) or with Lipofectamin 2000-siRNA max were cultured for $24 \mathrm{~h}$, and then the supernatant was discarded. MTT solution (5 mg/ml; Sigma-Aldrich; Merck KGaA, Darmstadt, Germany) was added into the cells and the cells were incubated for $5 \mathrm{~h}$ in $37^{\circ} \mathrm{C}$. The wave length of $490 \mathrm{~nm}$ was chosen to determine the optical density value of each well.

Apoptosis assay. Cells (1x105/well) was added into 96-well plate. The HL-7702 cells were cultured with siRNA-Lipofectamin 2000 mix for $6 \mathrm{~h}$ in $37^{\circ} \mathrm{C}$ and then the medium was discarded. The cells subsequently continued to be cultured in the serum-free medium for $24 \mathrm{~h}$. The binding buffer,
Table I. Primer sequences for qPCR analysis.

\begin{tabular}{|c|c|}
\hline Gene & Primer sequences $\left(5^{\prime} \rightarrow 3^{\prime}\right)$ \\
\hline Human Bcl-2 & $\begin{array}{l}\text { Positive sequence: GTGGCC } \\
\text { TTCTTTGAGTTCGG } \\
\text { Reverse sequence: GGCCGT } \\
\text { ACAGTTCCACAAAG }\end{array}$ \\
\hline Human Bax & $\begin{array}{l}\text { Positive sequence: ATGAAG } \\
\text { ACAGGGGCCCTTTT } \\
\text { Reverse sequence: GCAATC } \\
\text { ATCCTCTGCAGCTC }\end{array}$ \\
\hline Human caspase-3 & $\begin{array}{l}\text { Positive sequence: ACTGGA } \\
\text { CTGTGGCATTGAGA } \\
\text { Reverse sequence: GCACAA } \\
\text { AGCGACTGGATGAA }\end{array}$ \\
\hline Human GAPDH & $\begin{array}{l}\text { Positive sequence: CCAGAA } \\
\text { CATCATCCCTGCCT } \\
\text { Reverse sequence: CCTGCT } \\
\text { TCACCACCTTCTTG }\end{array}$ \\
\hline Human UGCG siRNA & $\begin{array}{l}\text { Positive sequence: CGCGAA } \\
\text { UCCAUGACAAUAUTT } \\
\text { Reverse sequence: AUAUUG } \\
\text { UCAUGGAUUCGCGTT }\end{array}$ \\
\hline Negative control siRNA & $\begin{array}{l}\text { Positive sequence: GCGACG } \\
\text { AUCUGCCUAAGAUdTdT } \\
\text { Reverse sequence: AUCUUA } \\
\text { GGCAGAUCGUCGUCGCdTdT }\end{array}$ \\
\hline Human GCS & $\begin{array}{l}\text { Positive sequence: TTCATG } \\
\text { TGTCATTGCCTGGC } \\
\text { Reverse sequence: AGCGTA } \\
\text { ATCTGTAGCGACCA }\end{array}$ \\
\hline
\end{tabular}

GCS, glucosylceramide synthase; UGCG, UDP-glucose ceramide glucosyltransferase.

Annexin V-FITC, or PI (both from Nanjing KeyGen Biotech Co., Ltd., Nanjing, China) was added into each well and the reaction at room temperature was done for 5 min in dark, the intracellular fluorescence was observed by fluorescence microscopy (Nikon Corporation, Tokyo, Japan).

ELISA assay. The HL-7702 cells with a density of $1 \times 10^{6} / \mathrm{ml}$ were cultured by RPMI-1640 medium with $10 \%$ fetal bovine serum in culture box with the diameter of $6 \mathrm{~cm}$. The cells were treated with or without siRNA $(0.12 \mu \mathrm{mol} / \mathrm{l})$. After the transfection of siRNA into the cells for $24 \mathrm{~h}$, the cell cultural supernatant was collected and detected by ELISA (Nanjing Jiancheng Bioengineering Institute, Nanjing, China) method according to instructions. The $450 \mathrm{~nm}$ wavelength was employed to detect the absorbance of each hole and the concentrations of tumor necrosis factor (TNF) $\alpha$ and cytochrome $c$ were calculated.

$R T$-qPCR. Cells (1x105/well) were put into 24 -well plate with $1 \mathrm{ml}$ medium containing $10 \%$ fetal bovine serum. Total 
A
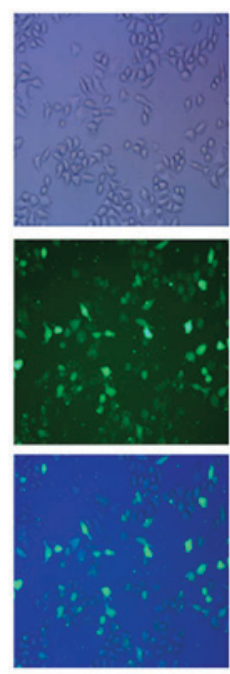

$1: 1\left(\mathrm{~V}_{\text {siRnA }} / \mathrm{V}_{\text {Lipo }}\right)$
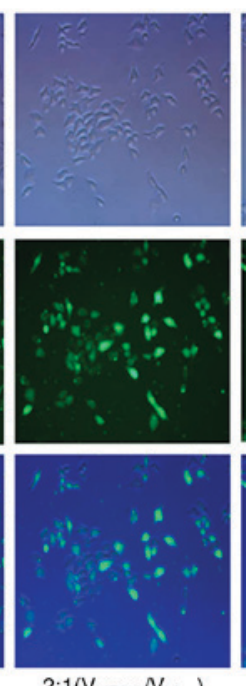

$2: 1\left(V_{\text {SiFNa }} / V_{\text {Lipo }}\right)$
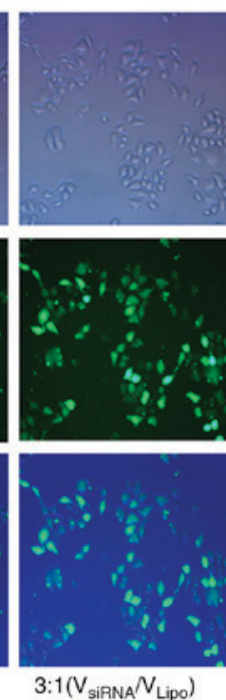

B

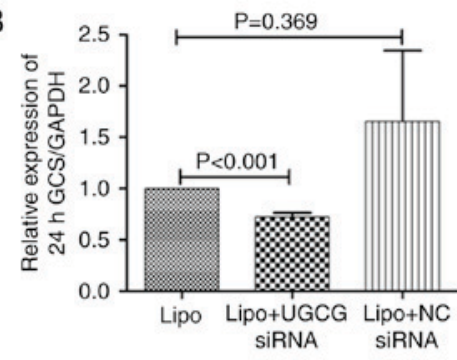

$(20 \times 10)$

Merge

$(20 \times 10)$

Figure 1. The transfection of siRNA and the effect of UGCG siRNA on the proliferation of HL 7702 (A). Fluorescence of hepatocytes after the transfection of siRNA with different concentrations (B). Inhibition effect of UGCG siRNA on GCS gene after transfection (C). Inhibition effect of UGCG siRNA on the proliferation of HL 7702. NC, negative control, Lipo, Lipofectamin 2000; UGCG, UDP-glucose ceramide glucosyltransferase; GCS glucosylceramide synthase.

RNA from hepatic cells were extracted using TRIzol lysate and chloroform on the ice after siRNA being transfected into the cells for $24 \mathrm{~h}$. The precipitation was formed at $-20^{\circ} \mathrm{C}$ for $2 \mathrm{~h}$ after centrifugation and UV spectrophotometry (Bio-Rad Laboratories, Inc., Hercules, CA, USA) using absorbance at 260 and $280 \mathrm{~nm}$ (A260/280) was used to determine the total RNA concentration and purity. And then the RNA was reversely transcripted into cDNA using the PrimeScript RT Reagent kit (Takara Bio, Inc., Otsu, Japan) according to the manufacturer's protocol. Real-time PCR amplification was carried out by the SYBR Green-based PCR Master Mix (Takara Bio, Inc.), which was used for the detection of mRNA expression. Gene expression levels were calculated after normalization to GAPDH. The $\Delta \Delta \mathrm{Ct}$ was calculated as follows: $\Delta \mathrm{Ct}_{\text {treated }}-\Delta \mathrm{Ct}_{\text {control }}$. And $2^{-\Delta \Delta \mathrm{Ct}}$ was calculated to represent the relative mRNA expression of target genes. The sequences of primers are presented in Table I.

Western blot analysis. The HL-7702 cells with a density of $3.0-3.5 \times 10^{5} / \mathrm{ml}$ were cultured in $6 \mathrm{~cm}$ diameter tissue culture dish (Corning Incorporated) overnight, and the supernatant was removed. The cells were treated with or without siRNA $(0.12 \mu \mathrm{mol} / \mathrm{l})$, the cells were cultured for $6 \mathrm{~h}$ and then were cultured in serum-free medium for $24 \mathrm{~h}$. The cell suspension was centrifuged by 2,000 rpm for 3-5 min, and were lysed by the RIPA with PMSF (RIPA:PMSF=100:1) on the ice for $10 \mathrm{~min}$, and then in centrifuge by $12,000 \mathrm{rpm}$ for 3-5 min. Bicinchoninic acid methods was employed for determination of protein concentration. Equivalent amounts of protein were subjected to polyacrylamide gel electrophoresis, followed by electroblotting onto PVDF membrane (Bio-Rad Laboratories, Inc.). PVDF membrane was incubated with primary antibody by 1:800 Caspase-3 antibody (Cell Signaling Technology, Inc., Danvers, MA, USA) at $4^{\circ} \mathrm{C}$ overnight. Then the PVDF was in 1:2,000 anti-rabbit antibody (Beijing Zhongshanjinqiao Co., Ltd., China), incubation for $1 \mathrm{~h}$ at room temperature, washed with TBST for 3 times. The protein on membranes was visualized by ECL western blotting kit (Thermo Fisher Scientific, Inc.) The densitometric analyses of images were performed using ImageJ software 1.46 (National Institutes of Health, Bethesda, MD, USA).

Statistical analysis. SPSS software version 19.0 (IBM Corp., Armonk, NY, USA) was used for data analysis. According to the data distribution type, the two groups of continuous variables were analyzed by independent sample $t$ test or Mann-Whitney test. $\mathrm{P}<0.05$ was statistically significant between the two groups.

\section{Results}

UGCG siRNA inhibits the GCS gene and the proliferation of hepatic cells. According to our transfection procession, the optimal concentrations of siRNA was $0.12 \mu \mathrm{mol} / 1\left(\mathrm{~V}_{\text {siRNA }} / \mathrm{V}_{\text {Lipo }}=3: 1\right.$, Fig. 1A), which showed higher transfection efficiency. HL-7702 cells were transfected by UGCG siRNA and negative control siRNA for $24 \mathrm{~h}$. The PCR results showed that compared with the transfection reagent group, the expression of GCS gene was significantly suppressed by UGCG siRNA $(\mathrm{P}<0.05)$, while the expression of GCS gene did not change significantly in negative control siRNA group ( $P>0.05$ ) (Fig. 1B). The results of MTT methods showed the proliferation of hepatic cells was significantly inhibited after the treatment of UGCG siRNA $(\mathrm{P}<0.05)$ compared with the transfection reagent (Fig. 1C).

Hepatic cells undergo apoptosis following UGCG siRNA treatment. The results showed that hepatic cells had apoptosis phenomenon, especially late apoptosis was obvious, but compared with the transfection reagent Lipofectamin 2000, the early and late apoptosis of liver cells differed slightly significantly in UGCG siRNA transfection group (Fig. 2). 

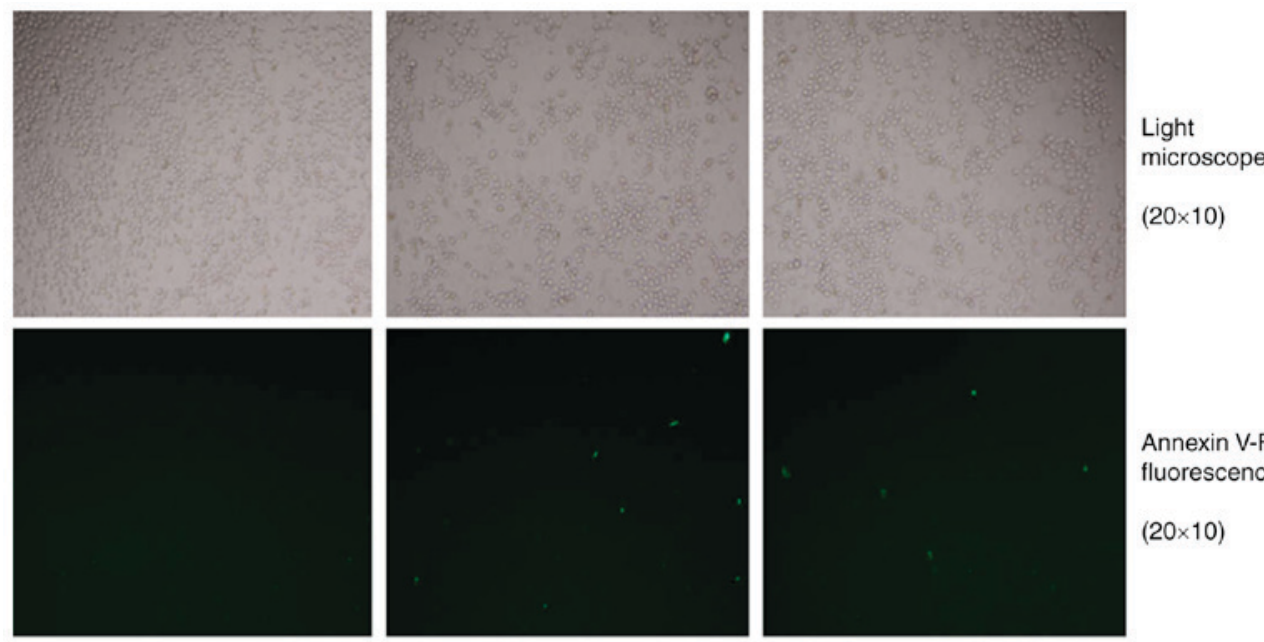

$(20 \times 10)$
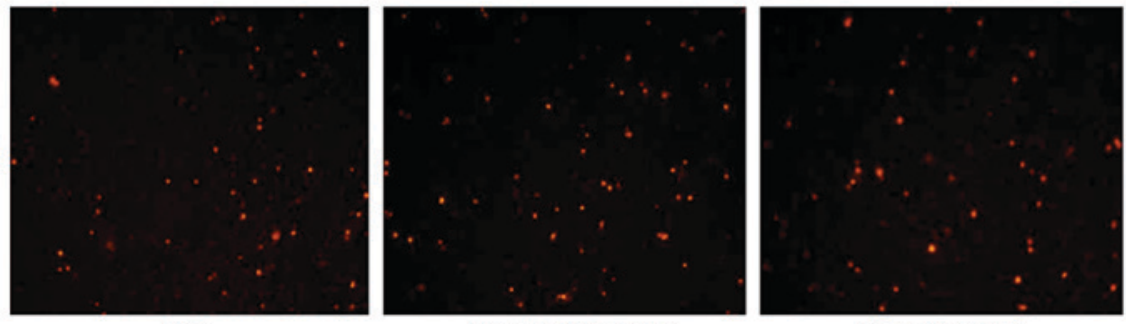

Annexin V-FITC

fluorescence

$(20 \times 10)$

Lipo

Lipo+UGCG siRNA

Lipo+NC siRNA

Figure 2. The fluorescence of HL 7702 hepatocytes after UGCG siRNA treatment. The Annexin V-FITC fluorescence signal is green and the PI fluorescence signal is red under fluorescence microscope, which shows the early and late apoptosis of liver cells. NC, negative control; Lipo, Lipofectamin 2000; UGCG, UDP-glucose ceramide glucosyltransferase.
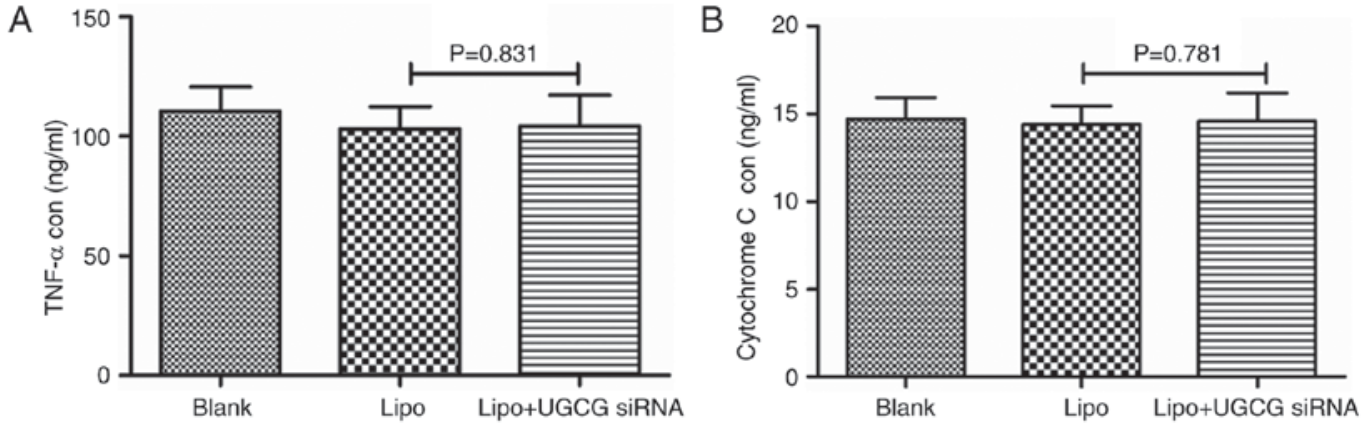

Figure 3. The concentration of (A) TNF $\alpha$ and (B) cytochrome $c$ in different groups. TNF $\alpha$, tumor necrosis factor $\alpha$; Lipo, Lipofectamin 2000; UGCG, UDP-glucose ceramide glucosyltransferase.

UGCG siRNA exhibits no effect on the secretion of TNF $\alpha$ and cytochrome $c$. In order to observe the expression of GCS gene on the secretion of TNF $\alpha$ and cytochrome $c$, the concentrations of TNF $\alpha$ and cytochrome $c$ in cell culture supernatant were detected by ELISA after the treatment of UGCG siRNA for $24 \mathrm{~h}$. The results showed the concentrations of TNF $\alpha$ and cytochrome $c$ did not change significantly between UGCG siRNA group and the transfection reagent Lipofectamin 2000 ( $\mathrm{P}>0.05$ ) (Fig. 3).

UGCG SiRNA regulates the expression of key apoptosis related-molecules in hepatic cells. In order to observe the effect of GCS on apoptosis signaling pathway in hepatic cells, we observed the gene expression of the key apoptosis related-molecules. The results showed that compared with the transfection reagent Lipofectamin 2000, the expression of Bcl-2 mRNA decreased ( $\mathrm{P}<0.05)$, Bax mRNA increased $(\mathrm{P}<0.05)$, the expression of caspase-3 mRNA also increased $(\mathrm{P}<0.05)$ in hepatic cells. As for the protein of caspase-3, we also observed that after transfection of UGCG siRNA for $24 \mathrm{~h}$, compared to transfection reagent group, the expression of caspase-3 protein upregulated in hepatic cells (Fig. 4).

\section{Discussion}

The present study is the first time to verify that the inhibition of expression of GCS gene may lead to the apoptosis of hepatic cells. In the design of this study, we used siRNA technology to target GCS gene expression, and on the basis of specific inhibition of GCS expression, our aims were to explore the effect 
A

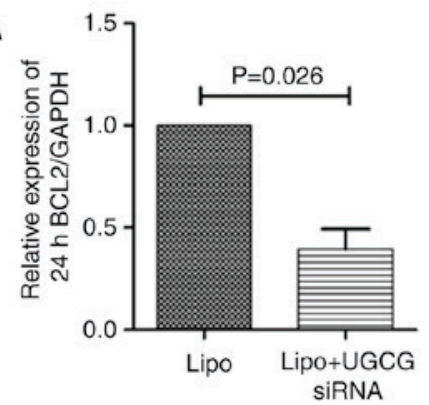

B

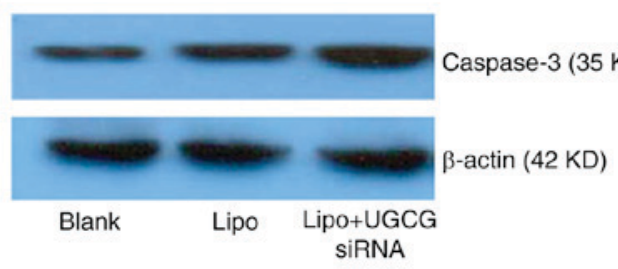

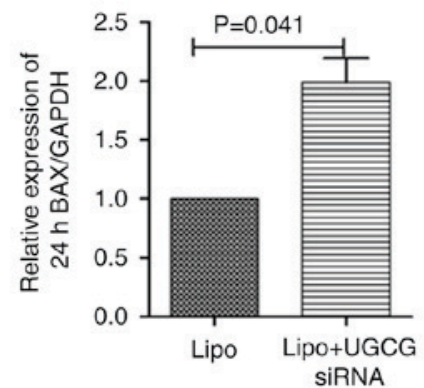
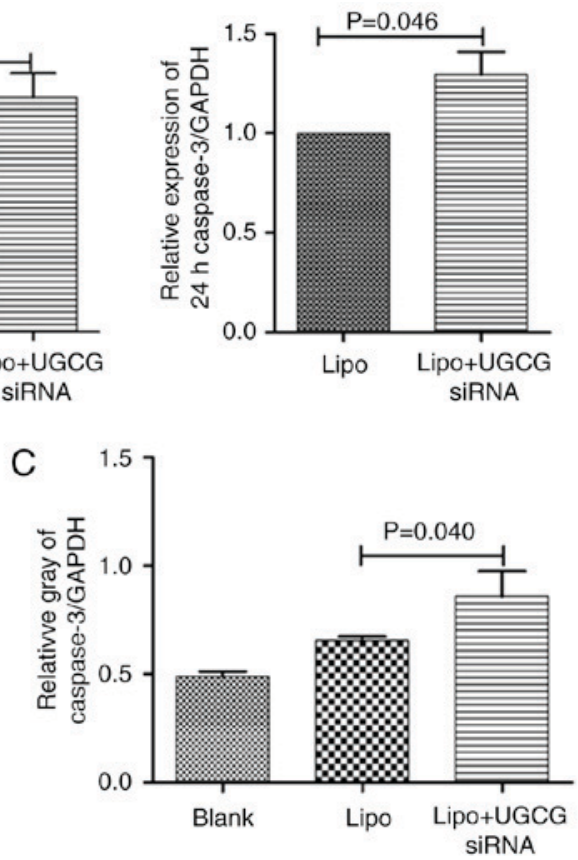

Figure 4. Expression of apoptosis-related genes and proteins in different groups. (A) Expression of Bcl-2, Bax, and caspase-3 genes in different groups; (B) Expression of caspase-3 proteins in different groups; (C) Relative gray of caspase-3 proteins in different groups. Lipo, Lipofectamin 2000; UGCG, UDP-glucose ceramide glucosyltransferase.

of GCS on the proliferation and apoptosis of hepatic cells and the possible mechanisms. Based on the present results, this study suggested that GCS might be involved in the cell cycle of hepatic cells.

In sphingolipid metabolism, GCS is the key enzyme catalyzing the glycosylation of ceramide, by regulating the balance of ceramide and glycosphingolipid to regulate the physiological activity of cells. During the glycosylation of ceramide, glucose is attached to the 1-hydroxyl group of ceramide to produce glucoseceramide $(13,14)$. GCS, which expresses in the eukaryotic cell membranes, is an intrinsic membrane protein encoded by the UGCG gene. The decreased expression of GCS leads to the decreased glycosylation of ceramide or elevated ceramide levels, and the elevated ceramide can trigger the endogenous or exogenous apoptosis. The main pathway for the regulation of cell apoptosis by ceramide activation is the endoplasmic reticulum and the mitochondrial pathways (15), which are involved in the TNF mediated- apoptosis pathway (16). The present study verified the targeting effect of UGCG siRNA sequence for GCS gene, which could inhibit the expression of GCS gene. The results showed that the proliferation of hepatic cells was obviously inhibited, and the cell activity of the hepatocytes after being transfected with UGCG siRNA obviously decreased. Followed by AnnexinV-FITC/PI double staining, the apoptosis of hepatic cells was also affected. Despite that the difference between the early and late apoptosis of hepatic cells was not significant, however, the trend of early and late apoptosis increased after the treatment of UGCG siRNA. This suggested that the proliferation or apoptosis of hepatic cells might be related to the inhibition of GCS, and the inhibition of the enzyme might lead to the increased ceramide levels, which contributed to the process of decreased activity of hepatocytes.

In order to study the mechanisms of GCS gene expression in the apoptosis of liver cells, the present study also observed the effect of UGCG siRNA on the Bcl-2 apoptosis pathway. As an anti-apoptotic effector, Bcl-2 could form a two-polymer with Bax to inhibit apoptosis, while Bax could increase the effect of Bcl-2 to promote apoptosis (17). It has been confirmed that the formation of Bax channels promotes the release of cytochrome $c$ and enter the cytoplasm, which can make the separation of Bcl-2 and Apaf1, and then activate caspase to induce apoptosis $(18,19)$. Our study found that after the inhibition of GCS gene expression, the upregulation of gene expression of Bcl-2 contributed to the decrease of Bax gene expression. In addition, caspase- 3 is the downstream of the caspase cascade, and is one of the most important proteases to perform the key function of apoptosis. The present study found that after the inhibition of GCS gene expression, the gene and protein expression of caspase-3 were both significantly increased. It might be due to the altered expression of apoptosis-related genes, which resulted in the execution of apoptosis effect.

TNF is involved in the proliferation and apoptosis of hepatocytes in the pathological processes of various liver diseases such as alcoholic liver disease, fulminant hepatitis, viral hepatitis and fatty hepatitis (20). The recent study showed that sphingomyelinase in sphingolipid metabolism was crucially involved in the regulation of TNF-induced liver cells death (21). Our study showed the extracellular concentration of TNF $\alpha$ did not change significantly after the treatment of UGCG siRNA. It was speculated that the apoptosis induced by GCS might not depend on the TNF $\alpha$. Moreover, it has been confirmed that the apoptotic pathways are divided into endogenous and exogenous ways (22). In the endogenous pathway, the permeabilization of mitochondrial membrane can lead to the release of cytochrome $c$ into the cytosol, and then interact with caspase to start a series of early apoptotic responses, the permeabilization and $\mathrm{Bcl}-2$ are involved in the mitochondrial 
membrane contributing to the release of cytochrome $c$ (23-25). In the present study, no changes of cytochrome $c$ were found in the groups. This might indirectly reflect the apoptosis induced by the endogenous expression of GCS gene had no relevance with cytochrome $c$-mediated endogenous apoptosis, however the further study is needed to verify the detailed mechanisms.

However, there are still issues to be addressed concerning other mechanisms which are not included in our study. The limitation of this study is paying no attention to other apoptotic related-pathways and shows no data about the effect of GCS on the apoptotic pathways in vivo. This is needed to be clarified in future.

In conclusion, the present study performed UGCG siRNA interference in vitro in the hepatic cells, and initially found that the decreased expression of GCS could contribute to the inhibition of proliferation and the increased apoptosis of hepatic cells, which was related to Bcl-2 mediated-apoptosis. Our study provided new clues for the role of GCS in the pathogenesis of apoptosis of hepatic cells. It will be needed to focus on the effect of abnormal metabolism of glycosphingolipid and other sphingolipid related-apoptosis pathway in the hepatic cells in the further research.

\section{Acknowledgements}

This work was supported by the Beijing Municipal Administration of Hospitals Ascent Plan (DFL20151601), the Fund of the First Hospital of Lanzhou University (ldyyyn2017-17) and the National Science and Technology Key Project on 'Major Infectious Diseases such as HIV/AIDS, Viral Hepatitis Prevention and Treatment' (2017ZX10202203-006, 2017ZX10302201-004, 2017ZX10203201-005, 2017ZX10201201).

\section{References}

1. Luedde T and Schwabe RF: NF- $\mathrm{BB}$ in the liver-linking injury, fibrosis and hepatocellular carcinoma. Nat Rev Gastroenterol Hepatol 8: 108-118, 2011.

2. Lee YA, Wallace MC and Friedman SL: Pathobiology of liver fibrosis: A translational success story. Gut 64: 830-841, 2015.

3. Heymann F and Tacke F: Immunology in the liver-from homeostasis to disease. Nat Rev Gastroenterol Hepatol 13: 88-110, 2016

4. Hannun YA and Obeid LM: Principles of bioactive lipid signalling: Lessons from sphingolipids. Nat Rev Mol Cell Biol 9: 139-150, 2008.

5. Nojima H, Freeman CM, Gulbins E and Lentsch AB: Sphingolipids in liver injury, repair and regeneration. Biol Chem 396 . 633-643, 2015.

6. Li JF, Qu F, Zheng SJ, Ren JY, Wu HL, Liu M, Liu H, Ren F, Chen Y, Zhang JL and Duan ZP: Plasma sphingolipids as potential indicators of hepatic necroinflammation in patients with chronic hepatitis $\mathrm{C}$ and normal alanine aminotransferase level. PLoS One 9: e95095, 2014.
7. Li JF, Qu F, Zheng SJ, Wu HL, Liu M, Liu S, Ren Y, Ren F, Chen Y, Duan ZP and Zhang JL: Elevated plasma sphingomyelin (d18:1/22:0) is closely related to hepatic steatosis in patients with chronic hepatitis C virus infection. Eur J Clin Microbiol Infect Dis 33: 1725-1732, 2014.

8. Li JF, Qu F, Zheng SJ, Ren F, Wu HL, Liu M, Ren JY, Chen Y, Duan ZP and Zhang JL: Plasma sphingolipids: Potential biomarkers for severe hepatic fibrosis in chronic hepatitis C. Mol Med Rep 12: 323-330, 2015.

9. Zheng SJ, Qu F, Li JF, Zhao J, Zhang JY, Liu M, Ren F, Chen Y, Zhang JL and Duan ZP: Serum sphingomyelin has potential to reflect hepatic injury in chronic hepatitis B virus infection. Int $\mathrm{J}$ Infect Dis 33: 149-155, 2015.

10. Zhang JY, Qu F, Li JF, Liu M, Ren F, Zhang JY, Bian DD, Chen Y, Duan ZP, Zhang JL and Zheng SJ: Up-regulation of plasma hexosylceramide (d18:1/18:1) contributes to genotype 2 virus replication in chronic hepatitis C: A 20 -year cohort study. Medicine (Baltimore) 95: e3773, 2016.

11. Garcia-Ruiz C, Morales A and Fernández-Checa JC: Glycosphingolipids and cell death: One aim, many ways. Apoptosis 20: 607-620, 2015.

12. Merrill AH Jr: Sphingolipid and glycosphingolipid metabolic pathways in the era of sphingolipidomics. Chem Rev 111: 6387-6422, 2011.

13. Liu YY, Hill RA and Li YT: Ceramide glycosylation catalyzed by glucosylceramide synthase and cancer drug resistance. Adv Cancer Res 117: 59-89, 2013.

14. Haynes CA, Allegood JC, Park H and Sullards MC: Sphingolipidomics: Methods for the comprehensive analysis of sphingolipids. J Chromatogr B Analyt Technol Biomed Life Sci 877: 2696-2708, 2009.

15. Morales A, Lee H, Goñi FM, Kolesnick R and Fernandez-Checa JC: Sphingolipids and cell death. Apoptosis 12: 923-939, 2007.

16. Verheij M, Bose R, Lin XH, Yao B, Jarvis WD, Grant S, Birrer MJ, Szabo E, Zon LI, Kyriakis JM, et al: Requirement for ceramide-initiated SAPK/JNK signalling in stress-induced apoptosis. Nature 380: 75-79, 1996.

17. Brooks C and Dong Z: Regulation of mitochondrial morphological dynamics during apoptosis by Bcl-2 family proteins: A key in Bak? Cell Cycle 6: 3043-3047, 2007.

18. Li P, Nijhawan D, Budihardjo I, Srinivasula SM, Ahmad M, Alnemri ES and Wang X: Cytochrome $c$ and dATP-dependent formation of Apaf-1/caspase-9 complex initiates an apoptotic protease cascade. Cell 91: 479-489, 1997.

19. Pan G, O'Rourke K and Dixit VM: Caspase-9, Bcl-XL, and Apaf-1 form a ternary complex. J Biol Chem 273: 5841-5845, 1998.

20. Mari M and Fernández-Checa JC: Sphingolipid signalling and liver diseases. Liver Int 27: 440-450, 2007.

21. García-Ruiz C, Colell A, Marí M, Morales A, Calvo M, Enrich C and Fernández-Checa JC: Defective TNF-alpha-mediated hepatocellular apoptosis and liver damage in acidic sphingomyelinase knockout mice. J Clin Invest 111: 197-208, 2003.

22. Elmore S: Apoptosis: A review of programmed cell death. Toxicol Pathol 35: 495-516, 2007.

23. Armstrong JS: Mitochondrial membrane permeabilization: The sine qua non for cell death. Bioessays 28: 253-260, 2006.

24. Nakagawa T, Shimizu S, Watanabe T, Yamaguchi O, Otsu K, Yamagata H, Inohara H, Kubo T and Tsujimoto Y: Cyclophilin D-dependent mitochondrial permeability transition regulates some necrotic but not apoptotic cell death. Nature 434: 652-658, 2005.

25. Gogvadze V, Orrenius S and Zhivotovsky B: Multiple pathways of cytochrome $c$ release from mitochondria in apoptosis. Biochim Biophys Acta 1757: 639-647, 2006. 